PROCEEDINGS OF THE AMERICAN MATHEMATICAL SOCIETY

Volume 124, Number 6, June 1996

\title{
GENERALIZED RATIONAL IDENTITIES OF SUBNORMAL SUBGROUPS OF SKEW FIELDS
}

\author{
KATSUO CHIBA
}

(Communicated by Ken Goodearl)

\begin{abstract}
Let $D$ be a skew field with infinite center $K$ such that $[D: K]=\infty$, and let $H$ be a non-central subnormal subgroup of the multiplicative group $D^{*}=D \backslash\{0\}$ of $D$. Then there are no non-trivial generalized rational identities of $H$. This generalizes a theorem proved by Makar-Limanov.
\end{abstract}

Several authors (e.g. [5], [6]) have studied subnormal subgroups of multiplicative groups of skew fields which satisfy some rational identity. In this note we shall study general rational identities of non-central subnormal subgroups of skew fields.

Let $D$ be a skew field with center $K$ and $K\langle X\rangle$ the free $K$-algebra on a finite set $X=\left\{x_{1}, x_{2}, \ldots, x_{n}\right\}$. We denote by $D\langle X\rangle=D *_{k} K\langle X\rangle$ the free product of $D$ and $K\langle X\rangle$ over $K$ and by $D(X)$ the universal skew field of fractions of $D\langle X\rangle$. Let $d=\left(d_{i}\right)$ be an element of $D^{n}$ and $\alpha_{d}: D\langle X\rangle \rightarrow D$ the $D$-ring homomorphism defined by $\alpha_{d}\left(x_{i}\right)=d_{i}, i=1,2, \ldots, n$. We denote by $\Sigma_{d}$ the set of all matrices over $D\langle X\rangle$ which are mapped by $\alpha_{d}$ to invertible matrices over $D$. Let $\Sigma_{d}^{-1}$ be the set of all entries of inverses $A^{-1}$ over $D(X)$ for all $A \in \Sigma_{d}$. Then $\Sigma_{d}^{-1}$ is a ring and it contains $D\langle X\rangle$ as a subring. Moreover, there is a $D$-ring homomorphism $\beta_{d}: \Sigma_{d}^{-1} \rightarrow D$ which extends $\alpha_{d}$ and satisfies that any element of $\Sigma_{d}^{-1}$ not in the kernel of $\beta_{d}$ has an inverse in $\Sigma_{d}^{-1}$ (see [4, Chapter 7]). Let $f=f\left(x_{i}\right)$ be an element of $D(X)$. If $f$ belongs to $\Sigma_{d}^{-1}$, we say $f$ is defined at $\left(d_{i}\right)$ and write $f\left(d_{i}\right)$ instead of $\beta_{d}(f)$.

The purpose of this note is to prove the following theorem which is a generalization of a theorem of Makar-Limanov [6, Theorem].

Theorem 1. Let $D$ be a skew field with infinite center $K$ such that the dimension of $D$ over $K$ is infinite, let $H$ be a non-central subnormal subgroup of the multiplicative group $D^{*}=D \backslash\{0\}$ of $D$ and let $f\left(x_{i}\right)$ be a non-zero element of $D(X)$. Then there is an element $\left(h_{i}\right) \in H^{n}$ such that $f\left(x_{i}\right)$ is defined at $\left(h_{i}\right)$ and $f\left(h_{i}\right) \neq 0$.

Let $R$ be a ring and $A$ an $m \times m$ matrix over $R$. We say that $A$ is non-full if there are matrices $P, Q$ over $R$ such that $A=P Q$ and $P$ is $m \times r, Q$ is $r \times m$, with $r<m$; in the contrary case $A$ is full. We denote by $e_{j}$ the column vector with 1 in the $j$ th place and 0's elsewhere and by $A_{j}^{*}$ the matrix obtained by replacing the 1st column of $A$ by $e_{j} \in{ }^{m} R$. We denote by $I_{k}$ the $k \times k$ identity matrix over $R$. Let $B$ and $C$ be matrices over $R$. If there are identity matrices $I_{p}$ and $I_{q}$ such that the diagonal sums $B \oplus I_{p}$ and $C \oplus I_{q}$ are associated, then $B$ and $C$ are said

Received by the editors June 7, 1994 and, in revised form, October 19, 1994.

1991 Mathematics Subject Classification. Primary 16R50; Secondary 16K40.

(C)1996 American Mathematical Society 
to be stably associated (see $[4$, p. 26]). If $a$ and $b$ are invertible elements of $R$, we denote by $(a, b)$ the multiplicative commutator $a^{-1} b^{-1} a b$ of $a$ and $b$. We denote by $R[t]$ the polynomial ring over $R$ in a central indeterminate $t, R[[t]]$ the ring of formal power series, and $R((t))$ the ring of formal Laurent series. There is a natural embedding of $R[[t]]$ into $R((t))$, so we shall think of $R[[t]]$ as a subring of $R((t))$. If $R$ is a semifir, we denote by $U(R)$ the universal skew field of fractions of $R$. Let $R$ and $S$ be $K$-algebras. We denote by $R *_{K} S$ the free product of $R$ and $S$ over $K$. Let $Y=\left\{y_{1}, y_{2}, \ldots, y_{n}\right\}$ be a finite set and $K\langle X, Y\rangle$ the free $K$-algebra on the set $X \cup Y=\left\{x_{1}, x_{2}, \ldots, x_{n}, y_{1}, y_{2}, \ldots, y_{n}\right\}$. We write $D\langle X, Y\rangle$ for $D *_{K}$ $K\langle X, Y\rangle$ and denote by $D(X, Y)$ the universal skew field of fractions of $D\langle X, Y\rangle$. We denote by $D\left\langle X, X^{-1}, Y, Y^{-1}\right\rangle$ (resp. $D\left\langle X, Y, Y^{-1}\right\rangle$ ) the $D$-subring of $D(X, Y)$ generated by $X \cup X^{-1} \cup Y \cup Y^{-1}=\left\{x_{1}, x_{2}, \ldots, x_{n}, x_{1}^{-1}, x_{2}^{-1}, \ldots, x_{n}^{-1}, y_{1}, y_{2}, \ldots, y_{n}\right.$, $\left.y_{1}^{-1}, y_{2}^{-1}, \ldots, y_{n}^{-1}\right\}$ (resp. $\left.X \cup Y \cup Y^{-1}\right)$.

To prove Theorem 1, we need several lemmas. We begin with the following.

Lemma 2. Let $R$ and $S$ be rings and let $\varphi: R \rightarrow S[[t]]$ be a ring homomorphism. Then there is a ring homomorphism $\tilde{\varphi}: R[[t]] \rightarrow S[[t]]$ such that $\tilde{\varphi}\left(\sum_{i \geq 0} r_{i} t^{i}\right)=$ $\sum_{i \geq 0} \varphi\left(r_{i}\right) t^{i}$, where $r_{i} \in R$ for all $i$.

Proof. Note that the sum $\sum_{i \geq 0} \varphi\left(r_{i}\right) t^{i}$ is defined in $S[[t]]$, as is every element of the form $\sum_{i \geq 0} a_{i} t^{i}$ with $a_{i} \in S[[t]]$. The fact that $\tilde{\varphi}$ is a ring map is then immediate from the corresponding property of $\varphi$.

Lemma 3. Let $F$ be a free group on the set $X \cup Y=\left\{x_{1}, x_{2}, \ldots, x_{n}, y_{1}, y_{2}, \ldots, y_{n}\right\}$, and $K[F]$ the group algebra over $K$. Then $D *_{K} K[F]$ is a fir and there is the natural isomorphism $\varphi: U\left(D *_{K} K[F]\right) \cong D(X, Y)$. In particular, $\varphi$ induces the isomorphism $D *_{K} K[S] \cong D\left\langle X, Y, Y^{-1}\right\rangle$, where $S$ denotes a subsemigroup of $F$ generated by $X \cup Y \cup Y^{-1} \subset F$ and $K[S]$ the semigroup algebra.

Proof. The result follows from [3, Theorem 5.3.2] and [2, Lemma 8].

Lemma 4. Let $h$ be a non-central element of $D$ and set $\bar{y}_{i}=y_{i}^{-1} h^{-1} y_{i} x_{i} y_{i}^{-1} h y_{i} \in$ $D\left\langle X, Y, Y^{-1}\right\rangle, i=1,2, \ldots, n$. Then the D-ring homomorphism $\tau$ of $D\langle X\rangle$ into $D\left\langle X, Y, Y^{-1}\right\rangle$ defined by $\tau\left(x_{i}\right)=\bar{y}_{i}-x_{i}, i=1,2, \ldots, n$, is injective.

Proof. First we show that the $D$-ring homomorphism $\alpha: D\langle X, Y\rangle \rightarrow D\left\langle X, Y, Y^{-1}\right\rangle$ defined by $\alpha\left(x_{i}\right)=x_{i}$ and $\alpha\left(y_{i}\right)=\bar{y}_{i}, i=1,2, \ldots, n$, is injective. Let $\Delta=\left\{d_{1}=\right.$ $\left.1, d_{2}=h^{-1}, d_{3}, \ldots\right\}$ be a $K$-basis of $D$ and $P$ the $K$-basis of $D\langle X\rangle$ consisting of the monomials $d_{i 0} x_{i 1} d_{i 1} x_{i 2} \ldots x_{i m} d_{i m}$ where $d_{i j} \in \Delta, x_{i j} \in X$. If $\alpha$ is not injective, let us take a non-zero element $f\left(x_{i}, y_{i}\right)$ of $\operatorname{ker} \alpha$ such that the $Y$-degree is minimal and write

$$
f\left(x_{i}, y_{i}\right)=\sum_{j, k} p_{j k}\left(x_{i}\right) y_{j} q_{j k}\left(x_{i}, y_{i}\right)+r\left(x_{i}\right)
$$

where $p_{j k}\left(x_{i}\right) \in P$ with $p_{j s}\left(x_{i}\right) \neq p_{j t}\left(x_{i}\right)$ for $s \neq t, q_{j k}\left(x_{i}, y_{i}\right) \in D\langle X, Y\rangle, r\left(x_{i}\right) \in$ $D\langle X\rangle$. Without loss of generality we may assume $p_{11}\left(x_{i}\right) y_{1} q_{11}\left(x_{i}, y_{i}\right) \neq 0$. Then we have

$$
\alpha\left(f\left(x_{i}, y_{i}\right)\right)=\sum_{j, k} p_{j k}\left(x_{i}\right) \bar{y}_{j} q_{j k}\left(x_{i}, \bar{y}_{i}\right)+r\left(x_{i}\right)=0 .
$$

Since each $p_{j k}\left(x_{i}\right) \bar{y}_{j} q_{j k}\left(x_{i}, \bar{y}_{i}\right)$ can be written as a $K$-linear combination of the monomials of the form $p_{j k}\left(x_{i}\right) y_{j}^{-1} h^{-1} y_{j} x_{j} u$, where $u \in D\left\langle X, Y, Y^{-1}\right\rangle$, by [1, Corollary 
8.1] and Lemma 3 we obtain $r\left(x_{i}\right)=0$. By [1, Corollary 8.1], $p_{j k}\left(x_{i}\right) y_{j}^{-1} h^{-1} y_{j} x_{j}$ 's are right $D\left\langle X, Y, Y^{-1}\right\rangle$-linearly independent, so we obtain $y_{1}^{-1} h y_{1} q_{11}\left(x_{i}, \bar{y}_{i}\right)=0$, and hence $q_{11}\left(x_{i}, \bar{y}_{i}\right)=0$, which contradicts the minimality of the $Y$-degree of $f\left(x_{i}, y_{i}\right)$. Thus $\alpha$ is injective. Now let $\beta: D\langle X\rangle \rightarrow D\langle X, Y\rangle$ be a $D$-ring homomorphism defined by $\beta\left(x_{i}\right)=y_{i}-x_{i}, i=1,2, \ldots, n$, and $\gamma: D\langle X, Y\rangle \rightarrow D\langle X\rangle$ a $D$-ring homomorphism defined by $\gamma\left(x_{i}\right)=x_{i}, \gamma\left(y_{i}\right)=2 x_{i}, i=1,2, \ldots, n$. Then $\gamma \beta=1$ on $D\langle X\rangle$. Hence $\beta$ is injective. Clearly, $\tau=\alpha \beta$, thus $\tau$ is injective. This completes the proof.

Lemma 5. Let $h$ be a non-central element of $D$ and $f\left(x_{i}\right)$ a non-zero element of $D(X)$ such that $f\left(x_{i}\right)$ is defined at $(1)=(1,1, \ldots, 1) \in D^{n}$. Then $f\left(x_{i}\right)$ is defined at $\left(\left(x_{i}, y_{i}^{-1} h y_{i}\right)\right)=\left(x_{i}^{-1} y_{i}^{-1} h^{-1} y_{i} x_{i} y_{i}^{-1} h y_{i}\right) \in D(X, Y)^{n}$ and $f\left(\left(x_{i}, y_{i}^{-1} h y_{i}\right)\right) \neq 0$.

Proof. We may assume that $f\left(x_{i}\right) \notin D$ and $f\left(x_{i}\right)$ is the first component of the solution $u$ of a matrix equation $A u=e_{j}$, where $A=A\left(x_{i}\right)$ is an $m \times m$ matrix over $D\langle X\rangle$ such that $A(1)$ is invertible over $D$ and $e_{j} \in{ }^{m} R$. Since $A(1)$ is invertible and $\left(x_{i}, y_{i}^{-1} h y_{i}\right)=1$ for $x_{i}=y_{i}=1, i=1,2, \ldots, n, A\left(\left(x_{i}, y_{i}^{-1} h y_{i}\right)\right)$ is a full matrix over $D\left\langle X, X^{-1}, Y, Y^{-1}\right\rangle$, hence invertible over $D(X, Y)$ by Lemma 3 . Thus $f\left(x_{i}\right)$ is defined at $\left(\left(x_{i}, y_{i}^{-1} h y_{i}\right)\right) \in D(X, Y)$. Suppose $f\left(\left(x_{i}, y_{i}^{-1} h y_{i}\right)\right)=0$. We write $A_{j}^{*}=A_{j}^{*}\left(x_{i}\right)$, the matrix obtained by replacing the 1st column of $A\left(x_{i}\right)$ by $e_{j} \in{ }^{m} R$. By Cramer's rule [4, Proposition 1.3, p. 384], $A_{j}^{*}\left(\left(x_{i}, y_{i}^{-1} h y_{i}\right)\right)$ is a singular matrix over $D(X, Y)$. Then, by Lemma $3, A_{j}^{*}\left(\left(x_{i}, y_{i}^{-1} h y_{i}\right)\right)$ is a non-full matrix over $D\left\langle X, X^{-1}, Y, Y^{-1}\right\rangle$. Hence $A_{j}^{*}\left(\left(1+x_{i} t, y_{i}^{-1} h y_{i}\right)\right)$ is a singular matrix over $D(X, Y)((t))$. Let us abbreviate $y_{i}^{-1} h^{-1} y_{i} x_{i} y_{i}^{-1} h y_{i}$ as $\bar{y}_{i}$ for $i=1,2, \ldots, n$. We have the following representation in $D(X, Y)((t))$

$$
\begin{aligned}
\left(1+x_{i} t, y_{i}^{-1} h y_{i}\right) & =\left(1+x_{i} t\right)^{-1} y_{i}^{-1} h^{-1} y_{i}\left(1+x_{i} t\right) y_{i}^{-1} h y_{i} \\
& =1+g_{i 1} t+g_{i 2} t^{2}+\cdots+g_{i p} t^{p}+\cdots
\end{aligned}
$$

where $g_{i 1}=\bar{y}_{i}-x_{i}, g_{i p} \in D\left\langle X, Y, Y^{-1}\right\rangle$. Since $A(1)$ is invertible, it follows that $A\left(\left(1+x_{i} t, y_{i}^{-1} h y_{i}\right)\right)=A\left(1+g_{i 1} t+g_{i 2} t^{2}+\cdots+g_{i p} t^{p}+\ldots\right)$ is an invertible matrix over $D(X, Y)((t))$. Thus $f\left(x_{i}\right)$ is defined at $\left(\left(1+x_{i} t, y_{i}^{-1} h y_{i}\right)\right) \in D(X, Y)((t))^{n}$ and $f\left(\left(1+x_{i} t, y_{i}^{-1} h y_{i}\right)\right)=0$. On the other hand, by [2, Lemma 7] $f\left(1+x_{i} t\right) \in D(X)((t))$ has the following form:

$$
f\left(1+x_{i} t\right)=f_{0}+f_{k}\left(x_{i}\right) t^{k}+f_{k+1}\left(x_{i}\right)^{k+1}+\ldots
$$

where $f_{0} \in D, k \geq 1$ and $f_{s}\left(x_{i}\right) \in D\langle X\rangle$ is homogeneous of $X$-degree $s$, for $s=k, k+1, k+2, \ldots$ and $f_{k}\left(x_{i}\right) \neq 0$. By Lemma 2 we have a $D$-ring homomorphism $\varphi: \quad D\langle X\rangle[[t]] \rightarrow D\left\langle X, Y, Y^{-1}\right\rangle[[t]]$ such that $\varphi(t)=t$ and $\varphi\left(x_{i}\right)=g_{i 1}+g_{i 2} t+$ $\cdots+g_{i p} t^{p-1}+\cdots, i=1,2, \ldots, n$. Then we have $\varphi\left(1+x_{i} t\right)=\left(1+x_{i} t, y_{i}^{-1} h y_{i}\right)$, $i=1,2, \ldots, n$, and

$$
\begin{aligned}
f\left(\left(1+x_{i} t, y_{i}^{-1} h y_{i}\right)\right) & =\varphi\left(f\left(1+x_{i} t\right)\right) \\
& =f_{0}+\varphi\left(f_{k}\left(x_{i}\right)\right) t^{k}+\varphi\left(f_{k+1}\left(x_{i}\right)\right) t^{k+1}+\cdots .
\end{aligned}
$$

The coefficient of $t^{k}$ of the right-hand side of $(1)$ is $f_{k}\left(\bar{y}_{i}-x_{i}\right)$. By Lemma 4 $f_{k}\left(\bar{y}_{i}-x_{i}\right) \neq 0$, hence $f\left(\left(1+x_{i} t, y_{i}^{-1} h y_{i}\right)\right) \neq 0$ in $D(X, Y)((t))$, a contradiction. This completes the proof. 
Lemma 6. Let $D$ be a skew field with infinite center $K$ and with the dimension of $D$ over $K$ infinite, and let $H$ be a non-central subnormal subgroup of $D^{*}=D \backslash\{0\}$. Then any full matrix over $D\langle X\rangle$ is invertible for some choice of $X$ in $H$.

Proof. Let $A\left(x_{i}\right)$ be an $m \times m$ full matrix over $D\langle X\rangle$ and $H$ a $r$-subnormal subgroup of $D^{*}$, that is, there is a chain $H=H_{r} \triangleleft H_{r-1} \triangleleft \cdots \triangleleft H_{0}=D^{*}$. We shall use induction on $r$. We denote by $\operatorname{diag}\left(x_{1}, x_{2}, \ldots, x_{n}\right)$ the $n \times n$ matrix such that the $(i, i)$-entry is $x_{i}$ and 0 's elsewhere. Since $A\left(x_{i}\right) \oplus \operatorname{diag}\left(x_{1}, x_{2}, \ldots, x_{n}\right)$ is a full matrix over $D\langle X\rangle$, by Cohn's specialization lemma [4, Lemma 5.9.5] there is an element $\left(d_{i}\right) \in D^{n}$ such that $A\left(d_{i}\right) \oplus \operatorname{diag}\left(d_{1}, d_{2}, \ldots, d_{n}\right)$ is invertible. Clearly $\left(d_{i}\right) \in H_{0}^{n}$ and $A\left(d_{i}\right)$ is invertible. Thus the lemma holds for the case $r=0$. Now let $s$ be the supremum of the rank of $A\left(x_{i}\right)$ as its arguments range over $H=H_{r}$, and let $h$ be a non-central element of $H_{r}$. Let $p\left(x_{i}\right)$ be a non-zero entry of $A\left(x_{i}\right)$. Then $p\left(x_{i}\right) \in D\langle X\rangle$ and so is defined at $(1) \in D^{n}$, and hence by Lemma $5, p\left(\left(x_{i}, y_{i}^{-1} h y_{i}\right)\right) \neq 0$. By Higman's trick (see [4, p. 272]), the $1 \times 1$ matrix $\left(p\left(\left(x_{i}, y_{i}^{-1} h y_{i}\right)\right)\right)$ is stably associated to a full matrix $P\left(x_{i}, y_{i}\right)$ over $D\langle X, Y\rangle$. By induction hypothesis there is an element $\left(a_{1}, a_{2}, \ldots, a_{n}, b_{1}, b_{2}, \ldots, b_{n}\right) \in H_{r-1}^{2 n}$ such that $P\left(a_{i}, b_{i}\right)$ is invertible. Hence $p\left(\left(a_{i}, b_{i}^{-1} h b_{i}\right)\right) \neq 0$ and $\left(a_{i}, b_{i}^{-1} h b_{i}\right) \in H_{r}^{n}$. Thus we may assume the rank of $A\left(u_{i}\right)$ is $s \geq 1$ for $\left(u_{i}\right) \in H_{r}^{n}$. We have to show that $s=m$. So let us assume that $s<m$. Applying the $D$-automorphism of $D\langle X\rangle$ defined by $x_{i} \rightarrow u_{i} x_{i}, i=2, \ldots, n$, we may assume that the rank of $A(1)$ is $s$ and using elementary transformations we may take the principal $s \times s$ minor of $A(1)$ to be invertible. Thus we can write

$$
A\left(x_{i}\right)=\left(\begin{array}{ll}
B_{1}\left(x_{i}\right) & B_{2}\left(x_{i}\right) \\
B_{3}\left(x_{i}\right) & B_{4}\left(x_{i}\right)
\end{array}\right)
$$

where $B_{1}\left(x_{i}\right)$ is $s \times s$ and $B_{1}(1)$ is invertible. Then we obtain

$$
\begin{aligned}
& \left(\begin{array}{cc}
1 & B_{1}\left(x_{i}\right)^{-1} B_{2}\left(x_{i}\right) \\
0 & B_{4}\left(x_{i}\right)-B_{3}\left(x_{i}\right) B_{1}\left(x_{i}\right)^{-1} B_{2}\left(x_{i}\right)
\end{array}\right) \\
& \quad=\left(\begin{array}{cc}
1 & 0 \\
-B_{3}\left(x_{i}\right) & 1
\end{array}\right)\left(\begin{array}{cc}
B_{1}\left(x_{i}\right)^{-1} & 0 \\
0 & 1
\end{array}\right)\left(\begin{array}{ll}
B_{1}\left(x_{i}\right) & B_{2}\left(x_{i}\right) \\
B_{3}\left(x_{i}\right) & B_{4}\left(x_{i}\right)
\end{array}\right) .
\end{aligned}
$$

Since $A\left(x_{i}\right)$ is invertible in $D(X), B_{4}\left(x_{i}\right)-B_{3}\left(x_{i}\right) B_{1}\left(x_{i}\right)^{-1} B_{2}\left(x_{i}\right) \neq 0$. Let $q\left(x_{i}\right) \in$ $D(X)$ be a non-zero entry of $B_{4}\left(x_{i}\right)-B_{3}\left(x_{i}\right) B_{1}\left(x_{i}\right)^{-1} B_{2}\left(x_{i}\right)$. Clearly $q\left(x_{i}\right)$ is defined at $(1) \in D^{n}$, hence by Lemma $5, q\left(x_{i}\right)$ is defined at $\left(\left(x_{i}, y_{i}^{-1} h y_{i}\right)\right) \in D(X, Y)^{n}$ and $q\left(\left(x_{i}, y_{i}^{-1} h y_{i}\right)\right) \neq 0$. Thus there is a full matrix $Q=Q\left(x_{i}, y_{i}\right)$ over $D\langle X, Y\rangle$ such that $q\left(\left(x_{i}, y_{i}^{-1} h y_{i}\right)\right)$ is the first component of the solution $u$ of a matrix equation $Q u=e_{j}$ and $Q_{j}^{*}=Q_{j}^{*}\left(x_{i}, y_{i}\right)$ is full. Clearly $B_{1}\left(\left(x_{i}, y_{i}^{-1} h y_{i}\right)\right)$ is a full matrix over $D\left\langle X, X^{-1}, Y, Y^{-1}\right\rangle$; by Higmans' trick, it is stably associated to a full matrix $B_{1}^{\prime}\left(x_{i}, y_{i}\right)$ over $D\langle X, Y\rangle$. By induction hypothesis there is an element $\left(v_{1}, v_{2}, \ldots, v_{n}, w_{1}, w_{2}, \ldots, w_{n}\right) \in H_{r-1}^{2 n}$ such that $B_{1}^{\prime}\left(v_{i}, w_{i}\right) \oplus Q\left(v_{i}, w_{i}\right) \oplus Q_{j}^{*}\left(v_{i}, w_{i}\right)$ is invertible. Hence $q\left(\left(v_{i}, w_{i}^{-1} h w_{i}\right)\right) \neq 0$ and the rank of $A\left(\left(v_{i}, w_{i}^{-1} h w_{i}\right)\right)$ is greater than or equal to $s+1$. Since $\left(\left(v_{i}, w_{i}^{-1} h w_{i}\right)\right) \in H_{r}^{n}$, this contradicts the definition of $s$ and the result follows.

Proof of Theorem 1. There is a full matrix $A\left(x_{i}\right)$ over $D\langle X\rangle$ such that $f\left(x_{i}\right)$ is the first component of the solution $u$ of a matrix equation $A u=e_{j}$ and $A_{j}^{*}=A_{j}^{*}\left(x_{i}\right)$ is full. Clearly $A\left(x_{i}\right) \oplus A_{j}^{*}\left(x_{i}\right)$ is full, so by Lemma 6 there is an element $\left(h_{i}\right) \in H^{n}$ 
such that $A\left(h_{i}\right) \oplus A_{j}^{*}\left(h_{i}\right)$ is invertible. Thus $f\left(x_{i}\right)$ is defined at $\left(h_{i}\right) \in H^{n}$ and $f\left(h_{i}\right) \neq 0$ by Cramer's rule. This completes the proof.

\section{REFERENCES}

1. G. M. Bergman, The diamond lemma for ring theory, Adv. in Math. 29 (1978), 178-218. MR 81b:16001

2. K. Chiba, Skew fields with a non-trivial generalized power central rational identity, Bull. Austral. Math. Soc. 49 (1994), 85-90. MR 95b:16017

3. P. M. Cohn, Skew field constructions, London Math. Soc. Lecture Notes Ser., vol. 27, Cambridge Univ. Press, Cambridge and New York, 1977. MR 57:3190

4. 87e: 16006

5. J. Z. Goncalves and A. Mandel, Are there free groups in division ring? Israel J. Math. 53 (1986), 69-80. MR 88a:16036

6. L. Makar-Limanov, On subnormal subgroups of skew fields, J. Algebra 114 (1988), 261-267. MR 89d:16024

Ninhama National College of Technology, Yagumo-Cho 7-1, Ninhama 792, Japan 